\title{
AVALIAÇÃO ESCOLAR: UM ESTUDO NO ENSINO DE CIÊNCIAS NATURAIS E SUAS ÁREAS
}

\author{
NATÁLIA DIAS \\ PALOMA MARQUES \\ LUCAS MESQUITA \\ CAROLINA DONÁ \\ MARILENA ROSALEN
}

\begin{abstract}
RESUMO
Este trabalho teve como objetivo realizar um estudo sobre a avaliação escolar do processo de aprendizagem em Ciências Naturais no Ensino Fundamental e nas suas áreas no Ensino Médio: Biologia, Física e Química. Por ser uma pesquisa do tipo estado da arte, ela tem um caráter bibliográfico, com intenção de mapear e discutir certa produção acadêmica na área do ensino de Ciências em diferentes épocas e locais. Diante disso, foi realizada uma busca nos trabalhos das atas das últimas cinco edições (2015, 2013, 2011, 2009, 2007) do Encontro Nacional de Pesquisa em Educação em Ciências (ENPEC), considerado o mais relevante evento na área de ensino de Ciências, do país, referentes à avaliação, em específico, trabalhos relacionados aos processos de ensino e aprendizagem, no ensino básico. Foi levantada uma amostra inicial de 105 trabalhos, do qual apenas 23 trabalhos foram selecionados, por se encaixarem nos critérios de inclusão e exclusão previamente estabelecidos nesta pesquisa. Como resultados principais, podemos destacar que: os trabalhos analisaram em sua maior parte o Ensino Médio, em especial o Ensino de Biologia; as abordagens metodológicas desses trabalhos foram equilibradas entre qualitativa, quantitativa e mista; observou-se que muitas das avaliações foram realizadas em aula, mas foram verificadas atividades do PIBID (Programa Institucional de Bolsa de Iniciação à Docência) e de projetos de extensão que foram utilizadas para a avaliação escolar; mesmo surgindo novos aspectos estruturais da avaliação, a pesquisa feita mostrou
\end{abstract}


que ainda predomina sua forma clássica e factual, priorizando a avaliação/exame de conteúdos conceituais.

Palavras-chave: Avaliação. Ensino de ciências naturais. Estado da arte.

\begin{abstract}
This work aims at the elaboration of a study about the school evaluation of the learning process in Natural Sciences in Elementary School and in its areas in High School: Biology, Physics and Chemistry. Because this study is a state of the art type, it has a bibliographic character, with the intention of mapping and discussing a certain academic production in the area of Science Teaching at different times and places. In the light of this, initially a search was made in the works of the last five editions (2015, 2013, 2011, 2009, 2007) of the National Meeting of Research in Science Education (ENPEC), considered the most relevant event in the area of Science Teaching, of the country, regarding the evaluation, in specific, works related to teaching and learning processes, in basic education. It collected an initial sample of 105 papers, of which only 23 papers were selected, as they fit the inclusion and exclusion criteria previously established in this research. As main results, we can highlight: the works analyze for the most part the High School, in particular the Teaching of Biology; the methodological approaches of these works are balanced between qualitative, quantitative and mixed; it was observed that the great majority of the evaluations were carried out in class, but there were activities of PIBID (Institutional Program of the Initiation Scholarship) and extension projects that were used for school evaluation; although new structural aspects of evaluation are emerging, it still predominates in its classic and factual form, prioritizing the evaluation / examination of conceptual contents.
\end{abstract} Key-words: Evaluation. Teaching of natural sciences. State of art.

\title{
INTRODUÇÃO
}

No Brasil, a sistematização dos exames escolares ocorreu nos séculos XVI e XVII, com base na atividade pedagógica dos padres jesuítas (séc. XVI) e pelo bispo protestante John Amós Comênio 
(séc. XVII). A obra publicada pelos jesuítas, Ratium Studiorum, regulamentava como conduzir os exames finais do educando.

Entre as décadas de 1960 e 1980, foi difundido um pensamento positivista sobre a avaliação no país como observado nas obras de Vitor Noll (1965); Medeiros (1974); Viana (1978); Turra (1973). Para Carvalho (2002), essas obras que eram tidas como manuais de construção de provas, com técnicas, planejamento, difundiram a influência do pensamento positivista, passando a subsidiar cursos de formação de educadores e uma legislação acerca da avaliação, integrando-se à orientação para a prática avaliativa nas escolas, de modo que as avaliações tornaram-se objetivas, assumindo uma função meramente classificatória, visando medir o sucesso ou o fracasso do aluno.

Publicações acadêmicas brasileiras a partir de 1980 começaram a tentar romper essa barreira, conforme afirma Calderón e Poltronieri (2013):

Fica evidenciado pelas pesquisas apresentadas que, nas décadas de 1970 e 1980, o paradigma tecnicista tinha grande força nas produções acadêmicas, evidenciando a visão positivista de avaliação. No decorrer da década de 1980, apesar do tecnicismo ainda exercer grande influência no campo da avaliação, observaram-se esforços no sentido de superar essa visão na direção de propostas mais formativas. É importante ressaltar que, nesse período, a grande preocupação dos pesquisadores era quanto à caracterização das práticas avaliativas na escola. Já na década de 1990, a preocupação dos pesquisadores deslocou-se das práticas avaliativas na Avaliação da aprendizagem na Educação Básica escola para o esclarecimento quanto às questões sobre ideias, concepções, teorias e pressupostos da avaliação (p. 890-891).

Na mesma direção, segundo Carvalho (2002), o aprofundamento do estudo sobre a temática fez surgir publicações com o intuito de discutir e aprofundar a abordagem qualitativa, não apenas para o processo de ensino e aprendizagem, mas também para a avaliação. Por exemplo, o livro de Maria Amélia Goldberg 
\& Clarilza Prado de Souza, publicado em 1979, "Avaliação de programas educacionais: vicissitudes, controvérsias, desafios". Iniciativas como essa foram ficando cada vez mais evidentes e concretizaram-se em obras de autores como Luckesi (1995), Depresbiteris (1999), Hoffmann (2000).

Segundo Armstrong (2008), a prática avaliativa estrutura-se de duas maneiras: discurso dos resultados acadêmicos, o qual encara a avaliação como examinatória e é uma prática educacional orientada por abordagens didáticas com diferentes metodologias com uma finalidade - treinar estudantes para obterem altas notas, e assim prepararem-se para os vestibulares; e a segunda é uma prática educacional orientada pelo desenvolvimento humano, que considera o educando como uma entidade viva, dotada de emoções, sentimentos, que não é inerte, com subjetividade, e que não se resume ao academicismo.

Segundo Luckesi (1999), a avaliação deveria ser diagnóstica, ou seja, ela deveria ser o instrumento dialético do avanço, instrumento da identificação de tomadas de decisão. Mas a avaliação nas escolas, geralmente, ocorre de maneira classificatória, ou seja, o julgamento de valor que serviria para uma tomada de decisão acaba servindo para classificar um ser humano histórico em um padrão determinado. A avaliação, cada vez mais, tem assumido a função de verificar, para atingir objetivos finais, sem levar em conta o processo educacional e de desenvolvimento humano dos estudantes.

Vivemos em uma época difícil para os educadores que acreditam na aprendizagem como algo que vale a pena buscar por si só e na formação humana dos alunos como propósito principal da escola. Resultados mais altos nos testes parecem estar na ordem do dia. A fim de atingir esse objetivo, os administradores esforçam-se em dar conta de agendas políticas; os professores ensinam seus alunos a obterem bons resultados nos testes; os alunos, por sua vez, ou reagem por meio da "cola" ou do uso de "esteroides de aprendizagem" (psicoestimulantes legais ou ilegais), ou simplesmente não se interessam pelas demandas impostas pela escola. A aventura da aprendizagem e o prazer 
de adquirir novas capacidades parecem ter sido abandonados ou seriamente reduzidos na sala de aula devido à inclinação ao atendimento de prazos, padrões de referências, ordens e objetivos (ARMSTRONG, 2008, p. 19).

Nessa perspectiva, a presente pesquisa teve por objetivo realizar um estudo dos trabalhos aceitos nos VI, VII, VIII, IX e X Encontro Nacional de Pesquisa em Educação em Ciências (ENPEC) para analisar de que modo se dão as práticas avaliativas, por esse assunto ser considerado uma lacuna na área. Para o alcance do objetivo, foram analisados trabalhos contidos nos anais do evento com a temática "Avaliação escolar na Educação em Ciências”, na educação básica.

A escolha do Encontro Nacional de Pesquisa em Educação em Ciências (ENPEC) foi pelo fato de este ser o principal evento em pesquisa no Ensino de Ciências, do país. Evento o qual reúne estudantes, pesquisadores e professores da área, com o intuito da divulgação científica e da promoção de discussões de propostas teórico-metodológicas e epistemológicas da pesquisa em educação. É um encontro de caráter nacional, sendo promovido pela Associação Brasileira de Pesquisa em Educação em Ciências (ABRAPEC), que foi fundada em 29 de novembro de 1997, após o primeiro ENPEC, como uma sociedade civil, de caráter científico e educacional, sem fins lucrativos e sem filiação político-partidária. Atualmente, a ABRAPEC tem por finalidade promover, divulgar e socializar a pesquisa em Educação em Ciências, por meio de encontros de pesquisa, escolas de formação para a pesquisa e publicações a respeito da pesquisa, bem como, atuar como órgão representante da área junto a entidades nacionais e internacionais de educação, pesquisa e fomento.

Segundo Salem e Kawamura (2013), a área de Ensino de Ciências tem uma trajetória ainda recente, porém é importante acompanhar seu desenvolvimento, no sentido de buscar identificar suas características e preocupações, especialmente no que diz respeito à interação entre as diversas áreas do conhecimento. 


\section{METODOLOGIA}

O presente trabalho aproxima-se do tipo estado da arte, assim denominado por Ferreira (2002) como de caráter bibliográfico, e por ter a intenção comum de mapear e discutir certa produção acadêmica, respondendo a aspectos e dimensões que vêm sendo destacados em diferentes épocas e locais.

Diante disso, inicialmente foi realizada uma busca nos trabalhos das atas das últimas cinco edições (2015, 2013, 2011, 2009, 2007) do Encontro Nacional de Pesquisa em Educação em Ciências (ENPEC) referentes à avaliação, em específico, trabalhos relacionados a processos de ensino e aprendizagem, no ensino básico. Com isso, tivemos um espaçamento temporal de produção de pesquisas de oito anos.

Para a busca, foi determinada como um descritor a palavra "Avaliação" contida no título dos trabalhos aceitos no encontro. Porém, nas edições VIII, IX e X do ENPEC, os trabalhos publicados nas atas do evento foram categorizados por meio de eixos temáticos, dessa forma, foram elencados nessas três últimas edições os trabalhos pertencentes à linha temática "Avaliação na Educação em Ciências".

Para fins de análise, foram incluídos os trabalhos que tratassem da avaliação no processo de ensino e aprendizagem, destes foram excluídos todos aqueles que não eram referentes à educação básica. A aplicação desses critérios deu-se mediante a leitura de todos os resumos e quando necessário foi realizada a leitura da versão completa do trabalho.

De acordo com a Tabela 1, verifica-se que foram encontrados 30 trabalhos que continham a palavra "Avaliação" no título no caso das edições VI e VII do ENPEC e 75 trabalhos presentes no eixo "avaliação na educação em ciências" no caso das edições VIII, IX e X do ENPEC, totalizando 105 trabalhos. 


\begin{tabular}{|l|l|l|}
\hline ENPEC & PERÍODO & NÚMERO DE ARTIGOS \\
\hline VI & 2006 & 18 \\
\hline VII & 2009 & 12 \\
\hline VIII & 2011 & 23 \\
\hline IX & 2013 & 21 \\
\hline X & 2015 & 31 \\
\hline & & 105 \\
TOTAL & & \\
\hline
\end{tabular}

Tabela 1: ENPEC's analisados, período e quantidade de trabalhos.

Fonte: Autoria própria, 2017.

A partir dos 105 trabalhos, buscou-se os que se referiam à avaliação do processo de ensino e aprendizagem na educação básica e se chegou a 23 trabalhos. Na sequência, foi realizada uma categorização dos 23 trabalhos selecionados, sendo que as categorias foram criadas de duas maneiras distintas, a primeira foi previamente estabelecida e a segunda foi criada por meio da leitura flutuante (BARDIN, 2009; MINAYO, 1991) das versões completas dos trabalhos.

As categorias preestabelecidas foram: tipo de pesquisa em avaliação escolar; tipo de conteúdo avaliado; tipo de avaliação; área temática. Quanto à categoria ancorada na tipologia de conteúdo, usamos os conteúdos conceituais, atitudinais e procedimentais (ZABALA, 2010). Para o autor, a identificação tipológica dos conteúdos que são trabalhados, é importante, pois permite analisar/conhecer aquilo que se trabalha, ou pretende trabalhar. Assim, os tipos de conteúdo a serem ensinados revelam, também, escolhas metodológicas, e isso se torna importante para a análise. Com relação aos tipos de pesquisas realizadas sobre o tema avaliação escolar, apoiamo-nos em Moreira (1988), que explicita em seu trabalho questões de pesquisa norteadoras relativas à temática que são: avaliação de conhecimento, atitudes e valores.

Os dados coletados por meio dessas categorias foram organizados em gráficos, de maneira que os resultados serão apre- 
sentados por meio da expressão da porcentagem das mesmas, fazendo assim uma análise quantitativa deles, a seguir. Optou-se pela construção de gráficos, pois as comparações das categorias teriam uma melhor visualização dos resultados, utilizando assim uma escala de medida intervalar.

\section{RESULTADOS E DISCUSSÃO}

Ancorando-se na análise documental, quantitativa, exploratório-descritiva retrospectiva, descrita por Lino et al. (2010), estruturamos nossa investigação dos 23 trabalhos sobre avaliação do processo de ensino e aprendizagem de Ciências e suas áreas, na educação básica, publicados nas atas dos respectivos ENPEC's analisados. Para a apresentação dos resultados e da discussão, utilizaremos as categorias já citadas: 1) tipo de pesquisa em avaliação escolar; 2) tipo de conteúdo avaliado; 3) tipo de avaliação; 4) área temática.

\section{Tipo de pesquisa em avaliação escolar}

Esta categoria foi organizada de modo a investigar dois eixos principais sobre as pesquisas em avaliação escolar, que foram publicadas nos ENPEC's: abordagem metodológica e questão norteadora desses trabalhos. Com relação às análises das metodologias, observou-se que entre os 23 trabalhos a distribuição do tipo de abordagem utilizada nas pesquisas foi bem nivelada, quanto ao seu tipo - Qualitativa, Quantitativa ou Mista. Para a classe qualitativa, obtiveram-se oito exemplares, para a abordagem quantitativa, sete trabalhos, e para a mista oito trabalhos: É interessante essa equiparação quanto à tipologia da metodologia escolhida para as pesquisas em avaliação escolar, pois evidencia que os pesquisadores da temática "avaliação" não possuem uma abordagem que seja unanimidade. Pode-se levantar três hipóteses para a similariedade do uso das abordagens: 1) o fato de que as avaliações escolares são analisadas de forma a se quantificar acertos/erros; 2) mas também são vistas como um processo qualitativo, por influência de um novo pensamento sobre a avaliação, e um distanciamento da postura examinatória, focada em fatos; 3) e ainda, existem análises que utilizam dessas 
duas perspectivas, sem determinismos metodológicos, de modo que um método responda/complete/corrobore os dados obtidos pelo outro, em suas unidades de análise, da aprendizagem em questão.

\section{Gráfico 1}

\section{Distribuição pelo tipo de Abordagem Metodológica}

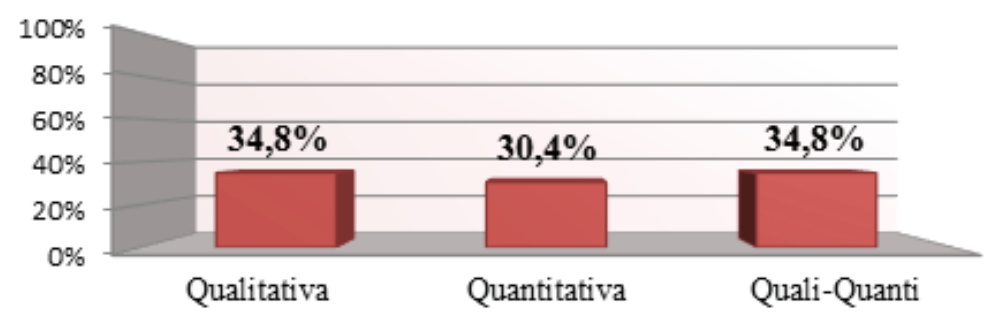

(Fonte: Autoria própria, 2017)

Quando procurou-se entender quais eram os objetivos de análise das avaliações, verificou-se que o avaliador buscava investigar sobre procedimentos, conhecimentos, valores, atitudes, ou ainda a união destes:

\section{Gráfico 2}

\section{Distribuição por Questão Que Norteavam o Trabalho}

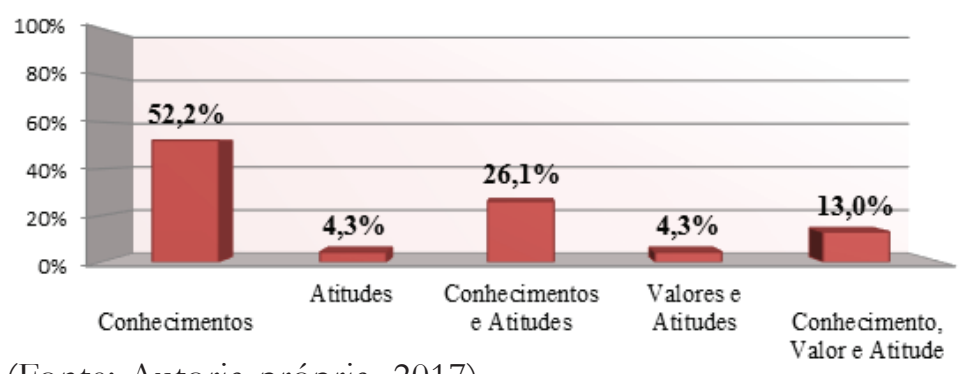

(Fonte: Autoria própria, 2017) 
Em relação às pesquisas observadas, a maioria buscava avaliação de conhecimento. Entre as possíveis pesquisas definidas por Moreira (1988), no presente trabalho, destacaram-se estudos que se caracterizavam por estudar como modelos alternativos de representação do conhecimento podem melhorar os procedimentos de avaliação, ou que métodos de avaliação têm mais fidedignidade e validade, e ainda como o uso de instrumentos heurísticos de aprendizagem pode enriquecer/melhorar os métodos de avaliação. Quanto à avaliação de atitudes, entre as categorias, foram identificados trabalhos que estudavam sobre a eficiência de questionários, de estratégias instrucionais que conduziam a ganhos positivos de atitude em longo prazo, e de como estratégias de interação aluno-professor podiam levar a ganhos positivos de atitudes em longo prazo. Por final, com relação a pesquisas que trabalhavam com valores, existia destaque para os pressupostos da relação entre compromissos epistemológicos e valores, procurando-os investigar (MOREIRA, 1988).

\section{Tipo de conteúdo avaliado}

Verificou-se que as questões norteadoras eram alicerçadas na avaliação de conceitos, visto que, 21 trabalhos (dado às categorias do gráfico a seguir temos que: Conceitual - 7 trabalhos, Conceitual e Procedimental - 5 trabalhos, Conceitual e atitudinal - 2 trabalhos, Conceitual, Procedimental e Atitudinal -7 trabalhos) encontram-se envolvidos com a categoria de conteúdo conceitual, e apenas 2 trabalhos estavam relacionados exclusivamente com avaliação de atitudes.

\section{Gráfico 3}

\section{Distribuição por Tipo de Conteúdo Avaliado}

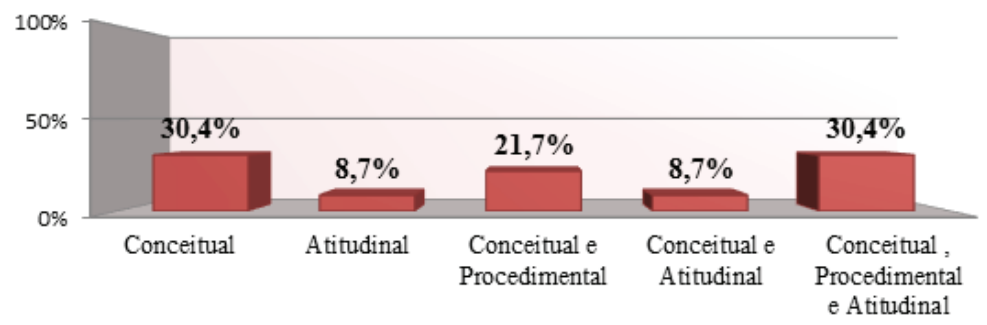

(Fonte: Autoria própria, 2017) 
Para Zabala (1998), os tipos de conteúdos podem servir de recursos para definir as diferentes posições sobre o papel que deve ter o ensino, dessa forma um ensino que apresenta diferentes tipos de conteúdo estará equilibrado, por sua vez, um ensino que defende a "propedêutica universitária", este focará em conteúdos conceituais.

Tem-se que as avaliações com foco em conteúdo conceitual eram centralizadas em analisar os príncipios e conceitos de determinado assunto em situações problemas ou factuais.

Já os conteúdos atitudinais, nos trabalhos analisados, foram encontrados em atividades avaliativas que utilizavam trabalhos em grupo e que contavam com um componente diferente, a afetividade, logo os conteúdos atitudinais sempre estavam atrelados a saberes experienciais do aluno e do professor.

O conteúdo procedimental, com relação aos artigos analisados, era utilizado e aplicado para a resolução de exercícios, construção de mapas conceituais e no processo de construção argumentativa.

\section{Tipo de Avaliação}

Durante a análise, procurou-se entender que perguntas de pesquisas nortearam os trabalhos recortados. Ademais, o que elas cobravam e quais seriam as alternativas para os testes clássicos focados em conteúdos conceituais. Somente cinco diferenciaram-se das noções clássicas de avaliação. Essas opções vinham em forma de mapas conceituais (NOVAK, 1980), influência teórica do construtivismo pedagógico e da incorporação deste na Teoria da Aprendizagem Significativa proposta por Ausubel (1963, 1968, 2000).

Com a avaliação clássica, estão as atividades propostas de forma factual, abertas ou fechadas, com o intuito de uma resolução de problema e aplicação de um conceito, por exemplo, muito utilizado em pesquisas no Ensino Médio, os cadernos de questões do Exame Nacional do Ensino Médio (ENEM).

A avaliação por argumentação está relacionada com a alfabetização científica e com o fato de os estudantes conseguirem construir argumentos, apropriando-se dos conceitos científicos, 
e assim, conseguirem, por exemplo, compreender um texto científico de determinado tema como a Anemia Faciforme, como apresentado por um trabalho, ou até mesmo na construção de um jornal de divulgação científica.

Os jogos foram englobados em dois trabalhos, sendo que um jogo era lúdico (conhecido como “Célula adentro”) e outro era digital (uso do Role Playing Game - RPG- para o Ensino de Química, com a temática de Combustíveis, em que os dados foram coletados durante a realização de um minicurso de 40 horas envolvendo alunos do primeiro ano do Ensino Médio). Os autores consideraram a utilização dessas ferramentas como alternativas para as avaliações clássicas, que poderiam ter um caráter quantitativo, e isolando-se em certos momentos, de uma avaliação como processo. Por fim, a avaliação Heurística encontrou-se no âmbito da usabilidade do $\mathrm{V}$ heurístico (GOWIN, 1981).

\section{Gráfico 4}

\section{Distribuição por Tipo de Avaliação}

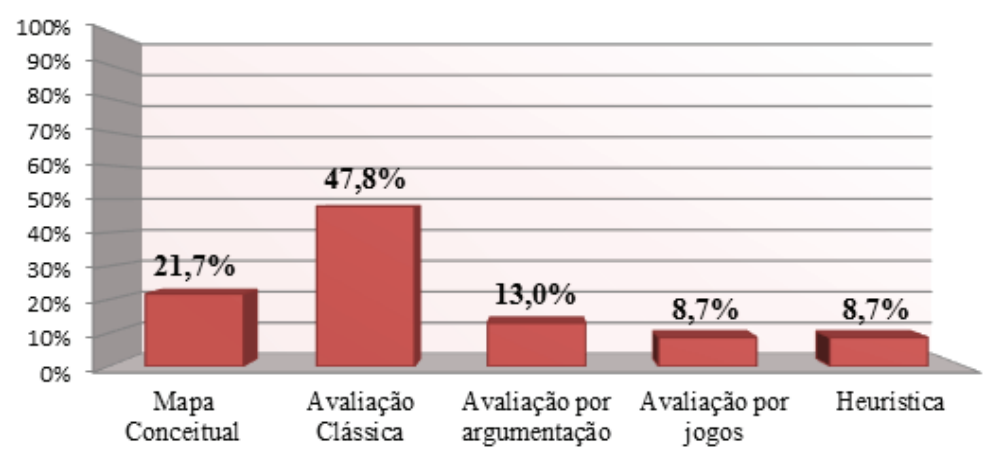

(Fonte: Autoria própria, 2017)

\section{4. Área temática}

Ao analisar os trabalhos sobre avaliação voltada para a educação básica, verificou-se que a maioria era voltada para o Ensino Médio. Do montante dos 23 trabalhos, 16 eram voltados 
ao Ensino Médio ou 69,6\%, Ensino Fundamental, 5 ou 21,7\%, ou para ambos, 2 ou $8,7 \%$ :

\section{Gráfico 5}

\section{Distribuição por Área de Ensino}

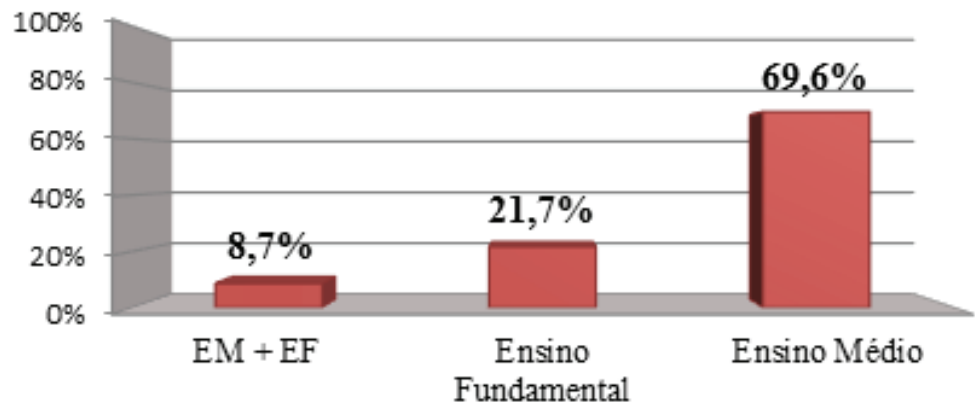

(Fonte: Autoria própria, 2017)

Entre os trabalhos do ensino médio, observou-se que um deles apresentou um diferencial - uma proposta de minicurso sobre entomologia para alunos do EM Técnico- Agrícola, em uma área rural do Paraná. É interessante, pois, é um caso em especial, o qual a cultura escolar, influenciou a escolha pelo conteúdo a ser trabalhado, e o tipo de curso influenciou na avaliação, o qual os estudantes elaborarão uma chave dicotômica - pelo curso abordar conteúdos da área de biológicas.

De acordo com os dados apresentados, a seguir observou-se que na educação básica o Ensino de Biologia tem despontado como a área que mais realiza pesquisas no tema, em comparação com as áreas de Física e Química, sendo que a temática Biologia apareceu, ao menos, em dez trabalhos, enquanto os outros apresentaram-se somente quatro e cinco vezes, respectivamente. Para Soares et al. (2006), a área de Ensino de Biologia - como campo de investigação, vem registrandro um crescimento notável, é um campo mais novo enquanto Ciência, perante as outras áreas (Química e Física), porém desde os anos 80, vem tendo uma crescente, por conta da consolidação da Biologia, e da preocupação dos pesquisadores da área com a educação científica 
desenvolvida no país (SLONGO, 2004). O tema Ciências Naturais foi abordado apenas duas vezes nos trabalhos analisados, um tratando de questões ambientais que permeavam as três áreas já citadas, sendo trabalhado no Ensino Fundamental, e o outro trabalho que ocorreu no Ensino Médio, de um estudo comparativo - entre uma escola pública e uma particular - de questões do Enem - também envolvendo as três áreas.

\section{Gráfico 6}

\section{Distribuição por Área da Ciência}

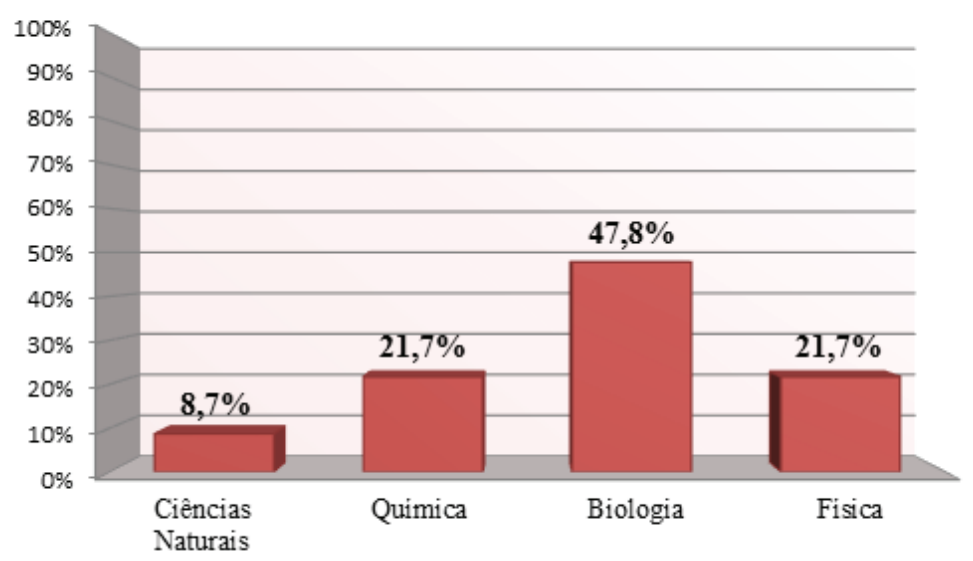

(Fonte: Autoria própria, 2017)

Outro dado interessante observado foi a utilização de atividades desenvolvidas pelo PIBID (Programa Institucional de Bolsa de Iniciação à Docência) e por projetos de extensão para a avaliação escolar. Embora em uma percentagem pequena, representa uma atitude de pertencimento dos projetos citados à comunidade escolar e uma tentativa de valorizar aprendizagens não mecanizadas: 


\section{Gráfico 7}

\section{Momento Pedagógico das Análises}

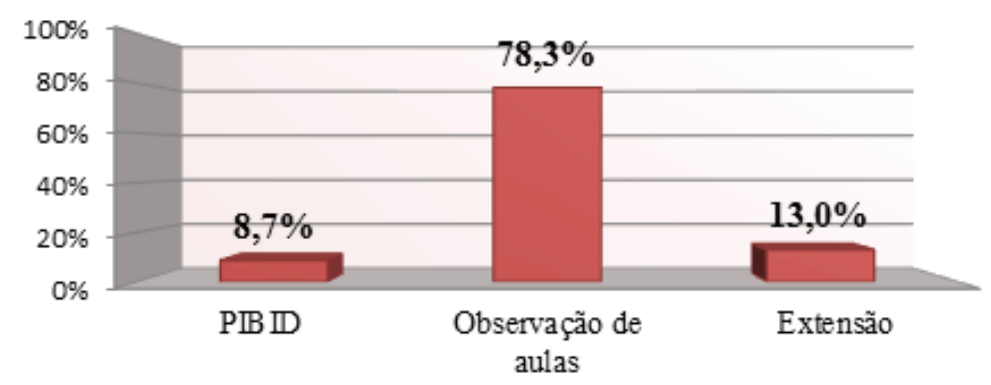

(Fonte: Autoria própria, 2017)

\section{CONSIDERAÇÕES FINAIS}

Com a análise dos 23 trabalhos selecionados, observou-se que a maioria dos trabalhos não priorizava as avaliações de atitudes e valores, dando maior preferência para as avaliações de conhecimento. Do total de trabalhos, apenas um utilizou tecnologia para auxiliar no processo avaliativo - um jogo de RPG. As propostas de avaliação com estratégias diferenciadas seguem certa tendência da aprendizagem significativa, tanto para o uso de Mapas Conceituais quanto para o uso de diagrama/V Heurístico.

Verificou-se que a avaliação escolar focava o Ensino Médio, talvez devido à realização do ENEM, nesse período, vez que algumas pesquisas de conhecimento usavam os cadernos de questões do Enem de Ciências Naturais como uma ferramenta de pesquisa.

Não se observou número alto de análises sobre ensino técnico, talvez uma grande necessidade, dada a nova proposta do Ensino Médio. Entre os trabalhos apresentados, apenas um era em ensino técnico em escola rural, mais um dado preocupante, que indica a falta de estudos nas regiões rurais.

Foi observado que as avaliações, no geral, mantiveram-se propícias ao seu formato clássico, com tendência pelo caráter examinátorio, momentâneo e não avaliativo processual da aprendizagem. 


\section{REFERÊNCIAS}

ARMSTRONG, Thomas. As melhores escolas: a prática educacional orientada pelo desenvolvimento humano. Porto Alegre: Artmed, 2008.

AUSUBEL, D. P. The psychology of meaningful verbal learning. New York: Grune \& Stratton, 255p. 1963.

Educational psychology: a cognitive view. New York: Holt, Rinehart and Winston, 685p. 1968.

. The acquisition and retention of knowledge: a cognitive view. Dordrecht: Kluwer Academic Publishers, 210p. 2000.

BARDIN L. Análise de conteúdo. Lisboa: Edições 70, 2009.

CALDERÓN, I. A. e POLTRONIERI, H. Avaliação da aprendizagem na Educação Básica: as pesquisas do estado da arte em questão (1980-2007). Rev. Diálogo Educ. Curitiba, v. 13, n. 40, p. 873-893, set./dez. 2013.

CARVAlHO, M.G. H. CARVALHO, M. A. Avaliação da aprendizagem: uma evolução histórica. Universidade Federal do Piauí. Ensino Médio e Educação Profissional, 2002.

DEPRESBITERIS, Léa. Avaliação de programas e avaliação de aprendizagem. In: METS, Lisa A.; FRANCO, Maria Laura P. Barbosa. Avaliação de Currículos e Programas: leituras complementares, 2. ed. Brasília: Universidade de Brasília, v. 4, n. 19, 1999.

FERREIRA, N. S. A. As pesquisas denominadas "estado da arte". Educação \& sociedade, v. 23, n. 79, p. 257-272, 2002.

GOLdBERG, Maria Amélia Azevedo; SOUZA, Clarilza Prado de. A Prática da avaliação. São Paulo: Cortez \& Moraes, 1979.

GOWIN, D. B. Educating. Ithaca, NY: Cornell University Press, 1981.

HOFfMAN, Jussara. Avaliação Mediadora. Uma Prática da Construção da Pré-escola a Universidade, 17. ed. Porto Alegre: Mediação, 2000.

LINO, M. M. et al. Perfil da produção científica e tecnológica dos grupos de pesquisa em educação em enfermagem da Região Sul do Brasil. Rev. Latino-Am. Enfermagem, v. 18, n. 3, p. 165-172, 2010.

LUCKESI, Cipriano Carlos. Avaliação da aprendizagem escolar. São Paulo: Cortez, 1995, 180p.

Avaliação da aprendizagem escolar, 9. ed. São Paulo: Cortez, 1999. MEDEIROS, E. B. Provas objetivas. Rio de Janeiro, Fundação Getúlio Vargas, 1974.

MINAYO, M. C. Interdisciplinaridade: uma questão que atravessa o saber, o poder e o mundo vivido. Medicina (Ribeiräo Preto), v. 24, n. 2, p. 70-7, 1991. MOREIRA, Marco Antônio. O professor-pesquisador como instrumento de melhoria do ensino de ciências. Aberto, Brasília, ano, v. 7, p. 42-53, 1988.

NOLL, V. Introdução às medidas educacionais. São Paulo: Pioneira, 1965. NOVAK, J. D. Uma teoria da educação. São Paulo: Pioneira. Trad. de M. A. Moreira. 252p. 1980.

SALEM, S., KAWAMURA, R. D. Ensino de ciências: Algumas características e tendências da pesquisa. Anais do IX Enpec. Águas de Lindoia/SP. 2013. 
SLONGO, I. I. P. A produção acadêmica em Ensino de Biologia. Florianópolis. Centro de Ciências da educação, Universidade Federal de Santa Catarina, 2004, 349s. (Tese de Doutorado).

SOARES, M. N., LABARCE, C., BONZANINI, K.T., CARVALHO, F. A., NARDI, R. Perspectivas atuais da pesquisa em ensino de Biologia. Anais do VI Enpec. Santa Catarina, 2006.

TURRA, C. M. Planejamento de ensino e avaliação. Porto Alegre: Ed. Emma, 1973.

VIANA, H. M. Testes em educação, São Paulo: IBRASA, 1978.

ZABALA, A. A prática educativa: Como ensinar. Porto Alegre: Artmed, 2010 .

\section{SOBRE OS AUTORES}

Natália Dias, Paloma Marques, Lucas Mesquita e Carolina Doná são mestrandos do PPG em Ensino de Ciências e Matemática/UNIFESP e Marilena Rosalen é docente do PPG em Ensino de Ciências e Matemática/UNIFESP.

E-mails de contato:

natalia.fdias@outlook.com;

palomam037@gmail.com;

lucasmesquita@hotmail.com;

cahdona@hotmail.com.

marilena.rosalen@gmail.com 